\title{
Outcomes of Complete Surgical Repair Versus Palliative Intervention in Neonates with Tetralogy of Fallot
}

\author{
Mohammed Hamzah ${ }^{1}$, Hasan Othman ${ }^{2}$, Krystel Chedid ${ }^{3}$, Mohammed Alsabri $^{4}$, Ibrahim \\ Qattea $^{5}$, and Hany $\mathrm{Aly}^{5}$ \\ ${ }^{1}$ Department of Pediatric Critical Care Cleveland Clinic Children's Cleveland OH \\ ${ }^{2}$ Michigan State University Department of Pediatrics and Human Development \\ ${ }^{3}$ Department of Pediatrics Cleveland Clinic Children's Cleveland $\mathrm{OH}$ \\ ${ }^{4}$ Brookdale University Hospital and Medical Center Department of Pediatrics \\ ${ }^{5}$ Department of Neonatology Cleveland Clinic Children's Cleveland $\mathrm{OH}$
}

December 18, 2021

\begin{abstract}
Background: Surgical management of symptomatic neonates with Tetralogy of Fallot (TOF) is controversial. Either primary surgical repair (EPSR) in neonates with TOF or a staged palliation with initial palliative intervention (PI). Aim: Compare outcomes of neonates with TOF who had EPSR and those who had PI. Materials and Methods: The study utilized the US National Inpatient Sample dataset for the years 2000 to 2018. Patients with EPSR and those with PI (aortic to pulmonary shunt or cardiac catheter palliative intervention) identified. Results: A total of 29,292 neonates with TOF were identified; of them 1726 neonates had EPSR, 4363 had PI. Hospital mortality was similar in both groups (PI 7.4\% vs EPSR 8.0\%, p = 0.41). Patient in the PI group had more comorbidities; chromosomal anomalies (PI 13.2\% vs. ESPR 7.8\%, $p<0.001$ ), prematurity (PI $15.1 \%$ vs. EPSR 10.4\%, $p<0.001$ ), and low birth weight $<2500$ grams (PI $15.4 \%$ vs. EPSR 10.3\%, $p<0.001$ ). Median length of stay and median cost of hospitalization were significantly higher in the EPSR (25 days vs. 19 days, and $\$ 312,405$ vs. $\$ 191,863$, respectively, $p<0.001)$. Conclusion: EPSR had similar mortality to PI but comes with a higher resource utilization and complications. If we include the cumulative morbidity and resource utilization associated with a two staged repair, EPSR could be proven as a better strategy in symptomatic neonates with TOF. A prospective superiority study on symptomatic neonates with TOF randomized to either ESPR or PI is needed to further answer this question.
\end{abstract}

Title:

Outcomes of Complete Surgical Repair Versus Palliative Intervention in Neonates with Tetralogy of Fallot

\section{Authors:}

Mohammed Hamzah, MDa ; Hasan F. Othman, MD ${ }^{\mathrm{b}}$; Krystel Chedid, $\mathrm{MD}^{\mathrm{c}}$, Mohammed Alsabri MD Ibrahim Qattea, $\mathrm{MD}^{\mathrm{e}}$, Hany Aly, MD

\section{Affiliations:}

a Department of Pediatric Critical Care, Cleveland Clinic Children's, Cleveland, OH

b Department of Pediatrics, Michigan State University/Sparrow Health System, Lansing, MI

c Department of Pediatrics, Cleveland Clinic Children's, Cleveland, OH

d Department of Pediatrics, Brookdale Hospital, Brooklyn, USA. 
e Department of Neonatology, Cleveland Clinic Children's, Cleveland, OH

Address correspondence to: Mohammed Hamzah, Department of Pediatric Critical Care, Cleveland Clinic Children's,

9500 Euclid Ave. M14, Cleveland, OH 44195, E-mail: HamzahM@ccf.org, Fax: (216) 444-3310, Telephone: (216) 444-0732

\section{Short title: Surgery versus Palliation in Neonatal Tetralogy}

Funding Source: No funding was secured for this study.

Financial Disclosure: All authors have no financial relationships relevant to this article to disclose.

Conflict of Interest: All authors have no conflicts of interest to disclose.

\section{Abbreviations:}

TOF: Tetralogy of Fallot

NIS: National Inpatient Sample

HCUP: Healthcare Cost and Utilization Project

AHRQ: Agency for Healthcare Research and Quality

ICD-9: International Classification of Disease-Ninths edition

ICD-10: International Classification of Disease-Tenths edition

LOS: length of stay

IQR: Inter Quartile Range.

Abstract

Background: Surgical management of severely symptomatic neonates with Tetralogy of Fallot (TOF) is controversial. Some centers perform primary surgical repair (EPSR) in neonates with TOF, while others perform staged palliation with initial palliative intervention (PI).

Aim: of the study: To compare outcomes between neonates with TOF who had EPSR and those who had PI.

Materials and Methods: The study utilized the US National Inpatient Sample dataset for the years 2000 to 2018. We identified patients who had EPSR and those with PI (aortic to pulmonary shunt or cardiac catheter palliative intervention).

Results: A total of 29,292 neonates with TOF were identified; of them 1726 neonates had EPSR, 4363 had PI. Hospital mortality was similar in both groups (PI $7.4 \%$ vs EPSR $8.0 \%, \mathrm{p}=0.41$ ). Patient in the PI group had more comorbidities; chromosomal anomalies (PI 13.2\% vs. ESPR 7.8\%, $p<0.001$ ), prematurity (PI $15.1 \%$ vs. EPSR $10.4 \%, p<0.001$ ), and low birth weight $<2500$ grams (PI $15.4 \%$ vs. EPSR $10.3 \%, p$ $<0.001)$. Median length of stay and median cost of hospitalization were significantly higher in the EPSR (25 days vs. 19 days, and $\$ 312,405$ vs. $\$ 191,863$, respectively, $p<0.001$ ).

Conclusion: EPSR had similar mortality to PI but comes with a higher resource utilization and complications. If we include the cumulative morbidity and resource utilization associated with a two staged repair, EPSR could be proven as a better strategy in symptomatic neonates with TOF. A prospective superiority study on symptomatic neonates with TOF randomized to either ESPR or PI is needed to further answer this question.

Introduction:

The ideal surgical approach when managing neonates with Tetralogy of Fallot (TOF) is still controversial (1). In 1945, subclavian artery to pulmonary artery anastamosis was created as a palliation for patients with TOF 
(2). A decade later, Lillehei and colleagues performed first intracardiac repair for TOF with ventricular septal defect closure and right ventricular outflow obstruction relief (3). The surgical and perfusion techniques, as well as the postoperative care has advanced and evolved since that time. Despite that evolution, the two concepts of surgical interventions had remained the same. There is still much debate on what is the ideal surgical approach to infants with TOF, an early primary surgical repair versus a two-staged surgical approach with an initial temporarily shunt for pulmonary blood flow. The aim of this study was to determine if one strategy had lower mortality than the other, and to assess the hospital length of stay and cost of charge in both groups.

Methods:

The data were obtained from the National (Nationwide) Inpatient Sample, part of the Healthcare Cost and Utilization Project (HCUP), sponsored by the Agency for Healthcare Research and Quality. This is the largest publicly available all-payer inpatient care database in the United States, containing data from more than seven million hospital stays each year (4). The National Inpatient Sample database randomly samples $20 \%$ of the discharges from participating hospitals in 47 US states and the District of Columbia. The sampling method provides a geographically distributed sample that represents all inpatient admissions in the nation. The use of data from approved public datasets is not considered human subject research; the study was granted exempt status from the Cleveland Clinic Institutional Review Board.

The study population was identified using the International Classification of Diseases, Ninth and Tenth Revisions, Clinical Modification (ICD-9\&10-CM). Data were queried from the years $2010-2018$. Neonates (age $<30$ days) with diagnosis of TOF were included in the study. These patients were further stratified into 3 groups: no intervention, complete surgical repair or palliative intervention (systemic-to-pulmonary arterial shunt, cardiac catheter intervention on the pulmonary valve or ductus arteriosus stent).

The primary outcome is the in-hospital mortality between the patients who underwent repair in the neonatal period versus those who underwent palliative intervention in the neonatal period. The secondary outcomes include the length of hospital stay and the hospital cost of charge.

Continuous variables were described using median and interquartile range (IQR). Categorical variables were described using frequencies and percentages. Demographics, clinical characteristics, and outcomes were compared using Mann-Whitney U test for continuous variables and Chi-square or Fisher's exact tests for categorical variables. Statistical significance was set at $\mathrm{P}<0.05$. The analysis was performed by SPSS software, version 25 (SPSS Inc., Chicago, IL) was used for statistical analysis.

Results:

We identified 29,292 neonates with TOF, neonates who needed intervention during first 30 days of life were found to be 6089 (20.78\%). Of those, $1726(28.35 \%)$ had early primary surgical repair (EPSR) and 4363 $(71.65 \%)$ had palliative intervention (PI). Table 1 shows basic characteristics of the study groups. The majority of these patients were males, PI male (55.0\%) and EPSR male $(57.3 \%)$. Neonates with PI had higher frequency of diagnoses chromosomal abnormalities (PI 13.2\% vs EPSR 7.8\%, p < 0.001). Other noncardiac anomalies were present at higher frequency in EPSR group (EPSR $21.9 \%$ vs. PI $19.2 \% \mathrm{p}=0.015$ ). Patients who had PI had higher rate of prematurity ( $<37$ weeks-gestation: PI $15.1 \%$ vs EPSR $10.4 \%, \mathrm{p}<$ 0.001 ) and higher frequency of low birth weight (birth weight $<2500$ grams: PI $15.4 \%$ vs EPSR $10.3 \%, \mathrm{p}<$ 0.001). Outcomes are presented in table 2. Hospital mortality was similar in both groups (PI 7.4\% vs EPSR $8.0 \%, \mathrm{p}=0.41$ ). Mechanical ventilation was used more frequently in the patients with EPSR (EPSR $50.4 \%$ vs PI $47.2 \%, \mathrm{p}=0.027$ ). The length of hospital stay was longer in the EPSR (EPSR 25 days vs PI 19 days, $\mathrm{P}<0.001$ ). The cost of charge was significantly higher in the EPSR (EPSR $\$ 312,405$ vs PI $\$ 191,863$, p $<0.001$ ). Extracorporeal membrane oxygenation utilization was similar in both groups (EPSR $4.6 \%$ vs PI $3.5 \%, \mathrm{p}=0.053)$.

Discussion:

There is still much debate about management of symptomatic neonates with TOF, a two staged repair 
(initial stage of securing source of pulmonary blood flow followed by later stage of a full repair) versus an early (neonatal) primary surgical repair. This study goal was to compare the outcomes of these two approaches, we evaluated 6089 neonates with TOF who either had an early primary surgical repair (1726, $28.3 \%$ ) versus 4363 neonates $(71.65 \%$ ) who had a palliative intervention (aortopulmonary shunt, or cardiac catheter intervention on pulmonary valve or ductus arteriosus stenting). The major findings of this study are as follows: in hospital mortality is similar between the two groups (EPSR vs PI) but EPSR approach comes with higher resource utilization (prolonged hospital stay, higher frequency of mechanical ventilation and higher cost of charge). It is important to mention that the resource utilization includes only the index hospitalization, however a two staged approach requires another hospitalization for full repair, therefore the cumulative morbidity and mortality of PI could be higher, but this was not the focus of this study. There are advantages and disadvantages to each approach, an early primary surgical repair would restore normal cardiovascular anatomy and physiology, resolve chronic cyanosis and promote neurodevelopment during critical period of brain growth in early infancy. Early primary surgical repair would also relief the right ventricular afterload and allow for early myocardial remodeling. Early repair may also restore the normal development of pulmonary vasculature and alveologenesis in patients with diminished pulmonary perfusion (5). The advantages of a two staged repair are mainly avoiding potential organ damage with neonatal openheart surgery and allow for time to attain somatic growth and organ maturity prior to the utilization of CPB in the second stage. The risks associated with systemic to pulmonary shunts are well described and they include shunt thrombosis, pulmonary artery distortion, excess of volume load on the pulmonary vasculature and the left ventricle with potential pulmonary vasculature disease and congestive heart failure. (6-9) In this study we are comparing approaches in symptomatic neonates with TOF who needed an intervention during the 30 days of life, we are not comparing early primary surgical repair versus late surgical repair, previous studies had shown that primary surgical repair at later age in infancy in asymptomatic TOF patients carries less morbidity and mortality than neonatal surgical repair $(10,11)$.

We used data from a large multicenter national database that randomly samples $20 \%$ of the discharges from hospitals across the United States with both a large number of patients and a wide range of practice variations, this allowed us to evaluate the surgical management in neonates with TOF. An important finding on the current surgical practice of TOF is that only $28.3 \%$ of neonates had an early complete surgical repair, which eludes that majority of centers prefer palliative intervention rather than early surgical repair in neonates with TOF. We also noted a patient selection bias in this retrospective study, we find that hospitals in the United States are inclined to use a palliative intervention in patients with significant comorbidities as low birth weight, prematurity and chromosomal anomalies. The only comorbidity that was present at higher frequency in the EPSR was the presence of other non-cardiac anomalies.

Limitations: there are several limitations to this study, we were not able to assess long-term outcomes, and specifically, which group will have better neurological outcome and which group will have better right ventricular function. We used an administrative database, and the study relied on the ICD-9 and ICD-10 diagnosis and procedure codes for identifying the study population and the associated comorbidities. The benefits of using this database is the large sample size, some limitations are inherent to all retrospective studies using administrative databases. Incorrect or missing data may exist and the lack of validation of the data collected by chart review is a source of potential bias for errors. However, in a large study such as ours, the patients' volume likely offset these inaccuracies. Another limitation of our study is the restriction to the hospitalization period, and this prevented us from evaluating long term outcomes.

Conclusion: Early primary surgical repair of neonates with TOF had similar in hospital mortality to neonates with TOF who received palliative intervention as neonates. The resource utilization was much higher in the EPSR but this included only the index hospitalization, however a two staged approach requires another hospitalization for full repair, therefore the cumulative morbidity and mortality of PI could be higher. A future prospective randomized study with long-term follow up is needed to further answer if early neonatal surgical repair is the ideal approach for symptomatic neonates with TOF.

References: 
1. Kanter, K. R., Kogon, B. E., Kirshbom, P. M., \& Carlock, P. R. (2010). Symptomatic Neonatal Tetralogy of Fallot: Repair or Shunt?Annals of Thoracic Surgery , 89 (3), 858-863. https://doi.org/10.1016/j.athoracsur.2009.12.060

2. Blalock A, Taussig HB: The surgical treatment of malformations of the heart in which there is pulmonary stenosis pulmonary atresia. JAMA 1945;128:189-202.

3. Lillehei, C. Walton Ph.D., M.D.; Varco, Richard L. Ph.D., M.D.; Cohen, Morley Ph.D., M.D.; Warden, Herbert E. M.D.; Gott, Vincent L. M.D.; Dewall, Richard A. M.D.; Patton, Cecelia R.N.; Moller, James H. M.D. The First Open Heart Corrections Of Tetralogy Of Fallot, Annals Of Surgery: October 1986 - Volume 204 - Issue 4 - P 49

4. Agency for Healthcare Research and Quality. Overview of the National (Nationwide) Inpatient Sample (NIS). Available at https://www.hcupus.ahrq.gov/nisoverview.jsp Accessed 23 Sep 2020

5. Johnson RJ, Haworth SG. Pulmonary vascular and alveolar development in tetralogy of Fallot: a recommendation for early correction. Thorax . 1982;37(12):893-901. doi:10.1136/thx.37.12.893

6. Stanley PH, Chartrand C, Davignon A, et al. Palliative surgery in tetralogy of Fallot. Can. J. Surg. J. canadien de chirurgie. 1981;24:475-479.

7. Yuan SM, Shinfeld A, Raanani E. The Blalock-Taussig shunt. J. Cardiac. Surg. 2009;24:101-108.

8. Fermanis GG, Ekangaki AK, Salmon AP, et al. Twelve year experience with the modified BlalockTaussig shunt in neo- nates. Eur J Cardiothorac Surg 1992;6:586-9.

9. Gladman G, McCrindle BW, Williams WG, Freedom RM, Benson LN. The modified Blalock-Taussig shunt: clinical impact and morbidity in Fallot's tetralogy in the current era. J Thorac Cardiovasc Surg 1997;114:25-30

10. Loomba, R. S., Buelow, M. W., \& Woods, R. K. (2017, June 1). Complete Repair of Tetralogy of Fallot in the Neonatal Versus Non-neonatal Period: A Meta-analysis. Pediatric Cardiology , Vol. 38, pp. 893-901. https://doi.org/10.1007/s00246-017-1579-8

11. Ghimire, L. V., Chou, F. S., Devoe, C., \& Moon-Grady, A. (2020). Comparison of In-Hospital Outcomes When Repair of Tetralogy of Fallot Is in the Neonatal Period Versus in the Post-Neonatal Period.American Journal of Cardiology , 125 (1), 140-145. https://doi.org/10.1016/j.amjcard.2019.09.025

Table 1. Basic Characteristics of Tetralogy of Fallot in neonates.

\begin{tabular}{llll}
\hline Characteristic & $\begin{array}{l}\text { Early Primary Surgical } \\
\text { repair }(\mathrm{n}=1726)\end{array}$ & $\begin{array}{l}\text { Palliative Intervention }(\mathrm{n} \\
=4363)\end{array}$ & $P$ \\
\hline $\begin{array}{l}\text { Sex }(\text { male }) \\
\text { Chromosomal }\end{array}$ & $986(57.3)$ & $2401(55.0)$ & 0.110 \\
$\begin{array}{l}\text { anomalies } \\
\text { Non-cardiac anomalies }\end{array}$ & $375(7.8)$ & $577(13.2)$ & $<\mathbf{0 . 0 0 1}$ \\
$\begin{array}{l}\text { Prematurity }(<37 \\
\text { week) }\end{array}$ & $180(10.4)$ & $837(19.2)$ & $\mathbf{0 . 0 1 5}$ \\
$\begin{array}{l}\text { Low Birth Weight }(< \\
2500 \text { grams })\end{array}$ & $178(10.3)$ & $659(15.1)$ & $<\mathbf{0 . 0 0 1}$ \\
$\begin{array}{l}\text { Necrotizing } \\
\text { Enterocolitis }\end{array}$ & $54(3.1)$ & $673(15.4)$ & $<\mathbf{0 . 0 0 1}$ \\
\hline
\end{tabular}

Data are expressed in frequency (\%); except for ${ }^{a}$ data that expressed in median (interquartile range); Chisquare or Fisher's exact tests were used for analysis except for ${ }^{\mathrm{a}}$ data that expressed in median (interquartile range); Mann-Whitney U test was used for analysis.

Table 2: Outcomes of Tetralogy of Fallot in neonates. 


\begin{tabular}{llll}
\hline Outcome & $\begin{array}{l}\text { Early Primary Surgical } \\
\text { repair }(\mathrm{n}=1726)\end{array}$ & $\begin{array}{l}\text { Palliative Intervention }(\mathrm{n} \\
=4363)\end{array}$ & $P$ \\
\hline Mortality & $138(8.0)$ & $322(7.4)$ & 0.414 \\
Length of stay, days $^{\text {a }}$ & $25(15-49)$ & $19(10-40)$ & $<\mathbf{0 . 0 0 1}$ \\
Cost of charge, dollars $^{2}$ & $312,405(162,403-558,467)$ & $191,863(89,022-393,106)$ & $<\mathbf{0 . 0 0 1}$ \\
Mechanical ventilation & $870(50.4)$ & $2061(47.2)$ & $\mathbf{0 . 0 2 7}$ \\
ECMO & $79(4.6)$ & $153(3.5)$ & 0.053 \\
\hline
\end{tabular}

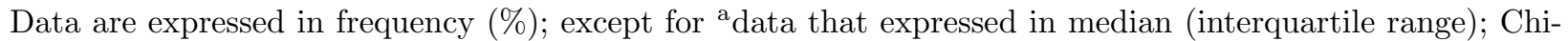
square or Fisher's exact tests were used for analysis except for ${ }^{\mathrm{a}}$ data that expressed in median (interquartile range); Mann-Whitney U test was used for analysis. 